\title{
Ultra low actuation voltage RF MEMS switch
}

\author{
Ali Attaran* and Rashid Rashidzadeh
}

\begin{abstract}
In this brief a new low actuation voltage RF MEMS switch is presented which can be integrated and controlled with available CMOS technologies. Despite the advantages in the design of RF MEMS switches designing a low actuation voltage RF MEMS switch is still a challenging task. To overcome this problem, a small size RF MEMS switch utilizing a moving plate with multiple holes supported by a low spring constant beam is presented in this work. Experimental measurement results indicate pull-in voltage of $0.5 \mathrm{~V}$ and lift-off voltage of $0.3 \mathrm{~V}$ for $1.5 \mu \mathrm{m}$ displacement. The measured return loss and insertion loss are better than $-20 \mathrm{~dB}$ and $-0.1 \mathrm{~dB}$ respectively for a frequency range extending from $3 \mathrm{kHz}$ to $3 \mathrm{GHz}$. The switching time is less than $0.22 \mathrm{~ms}$ when the switch is turned on with a CMOS buffer from TSMC-65 nm technology with $1.00 \mathrm{~V}$ supply voltage.
\end{abstract}

Keywords: Radio frequency micro-electro-mechanical-system (RF MEMS) switch; Low actuation voltage; Microfabrication

\section{Introduction}

Micro-Electro-Mechanical Systems (MEMS) devices have been vastly employed for many applications including Radio-Frequency (RF) components such as RF switches, variable capacitors and inductors [1-4]. RF and microwave MEMS switches have been subject of many research studies due to their superior performance such as high isolation, low insertion loss, good linearity and low power consumption. MEMS switches are either direct-contact style or capacitive type. Direct-contact MEMS switches are commonly used for application where the frequency of operation is limited to a few gigahertz. Capacitive switches are preferred for higher frequencies. To close or open MEMS switches the force is applied through electrostatic, magnetic fields or thermally induced forces [3]. RF MEMS switches mostly employ electrostatic actuation due to low power consumption and short switching time as well as compatibility with electrical circuits and components. Electrostatic MEMS switches require a relatively high actuation voltage to drive the movable part. For the purpose of wireless communication or integrated circuit design, it is highly desired to reduce the pull-in voltage of MEMS switches. Various design techniques are proposed in the literature

\footnotetext{
* Correspondence:

Ali1111@uwindsor.ca

Electrical and Computer Engineering Department, University of Windsor,

Windsor, Ontario, Canada
}

to reduce the actuation voltage. In [5] a pull-up type RF MEMS switch with low actuation voltage of $4.5 \mathrm{~V}$ is presented. In [6], a mechanically coupled low-voltage electrostatic resistive RF multithrow switch with $15 \mathrm{~V}$ is proposed.

In general, low actuation MEMS switches suffer from a range of problem including stiction, dielectric charging, hot switching life time and low power handling. In the proposed application in this work, a minute amount of power passes through the MEMS switch and thus the power handling and hot switching life time are not important issues. However, stiction and holding can affect the tag operation. The design of the MEMS switch has to be optimized to reduce these undesired effects.

In this work, a helix restoring spring is utilized to implement a low actuation voltage RF MEMS. The implemented switch includes a moving plate with multiple square holes that contribute to the faster operation of the switch by reducing the air damping factor under the moving plate. The dimple is added to reduce the gap between the movable plate and the output transition line to lowers the actuation voltage. A low-cost MEMS microfabrication process has been used to fabricate the MEMS switch. The implemented switch can be integrated with available $1.00 \mathrm{~V}$ supply CMOS technologies.
黑 Springer
(C) 2015 Attaran and Rashidzadeh. Open Access This article is distributed under the terms of the Creative Commons Attribution 4.0 International License (http://creativecommons.org/licenses/by/4.0/, which permits unrestricted use, distribution, and reproduction in any medium, provided you give appropriate credit to the original author(s) and the source, provide a link to the Creative Commons license, and indicate if changes were made. 


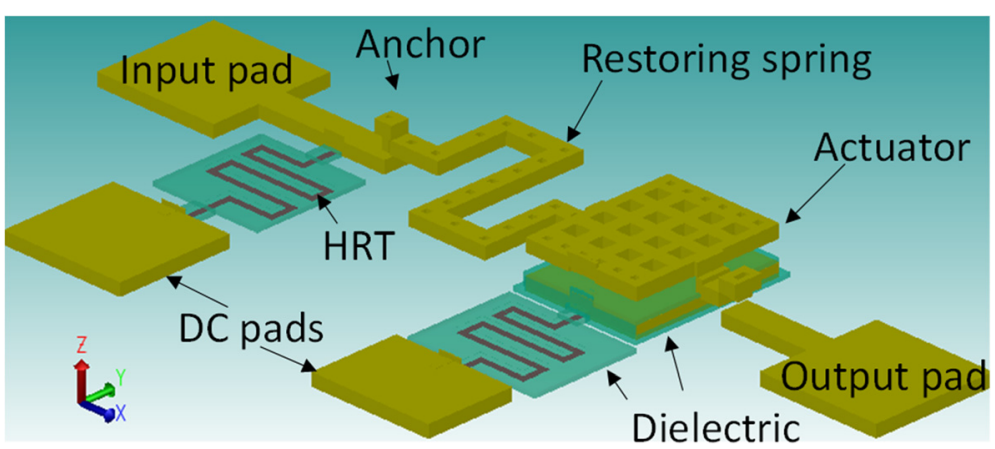

Fig. $13 \mathrm{D}$ view of the implemented low actuation voltage MEMS switch using Coventorware

\section{Mechanical and RF design principles}

There are different structures to implement a MEMS switch; among them cantilever switch supports a relatively low pull-in voltage $[3,4]$. The pull-in voltage, $V_{\text {pull-in }}$, of a MEMS switch can be calculated from Eq. (1) [4],

$$
V_{p u l l-i n}=\sqrt{\frac{8 K_{Z} g_{0}^{3}}{27 W \varepsilon_{0}}}
$$

where $K_{Z}$ is the spring constant, $g_{0}$ is the gap between metal plates, $W$ is the area of the pads and $\varepsilon_{0}$ is the air permittivity. The pull-in voltage, $V_{\text {pull-in }}$, can be reduced by (a) increasing the area of the pads, $W$. (b) reducing the gap between metal plates, $g_{0}$, and (c) reducing the spring constant $K_{Z}$. There is a trade-off between the size of the
MEMS switch and its pull-in voltage. To lower the pull-in voltage, the size of the MEMS has to be increased.

A MEMS switch are mainly implemented using either a fixed to fixed beam structure [3], or a cantilever beam actuator. Fixed to fixed MEMS switches as compared to cantilever switches support larger electrode areas and therefore larger actuation capacitances; whereas cantilever structures support lower pull-in voltages. The spring constant $K_{Z}$ in the $\mathrm{z}$-direction can be determined from Eq. (2) [3, 4].

$$
K_{z}=E W\left(\frac{t}{L_{c}}\right)^{3}\left\langle 1+\frac{L_{s}}{L_{c}}\left\{\left(\frac{L_{s}}{L_{c}}\right)^{2}+12(1+v)\left[1+\left(\frac{W}{t}\right)^{2}\right]^{-1}\right\}\right\rangle^{-1}
$$

where $E$ is Young's modulus, $v$ is Poisson's ratio of the metal. The lengths of the cantilever and spring are $L_{c}$
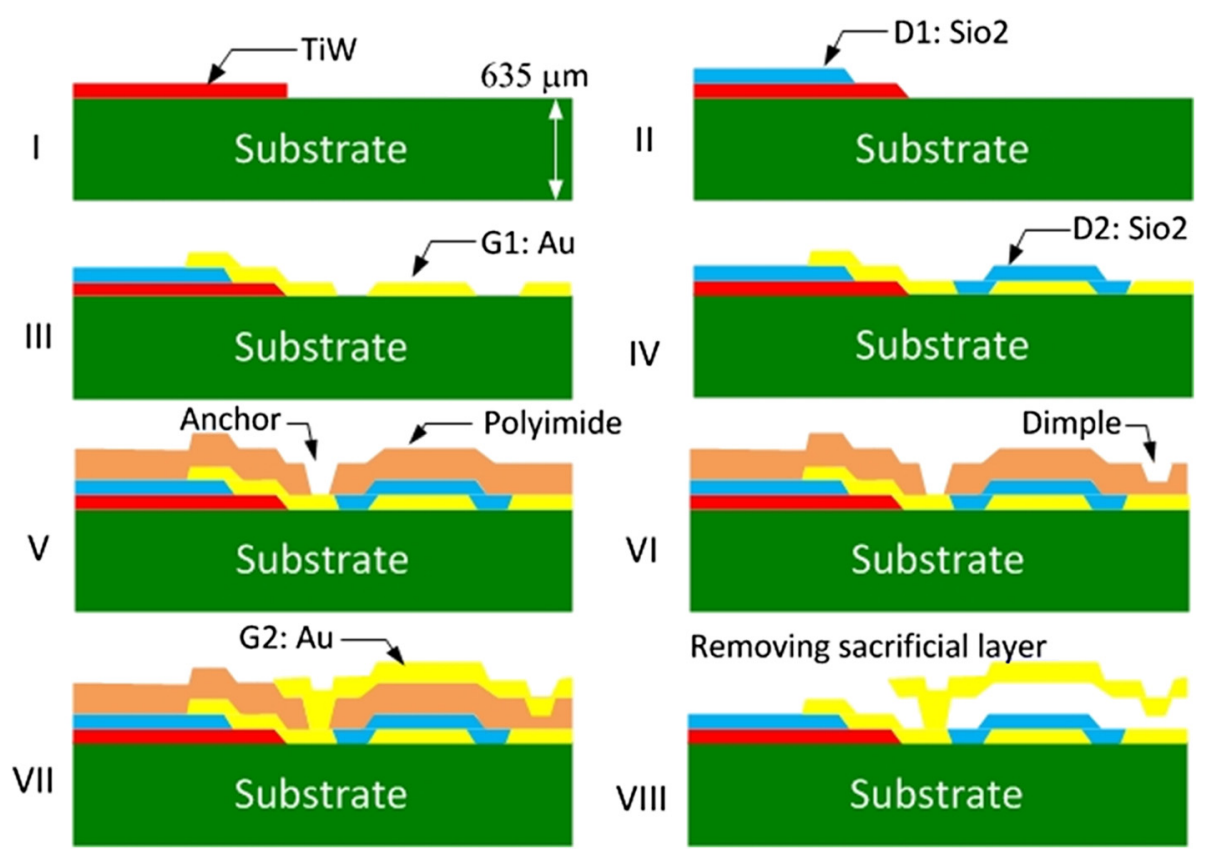

Removing sacrificial layer

VIII

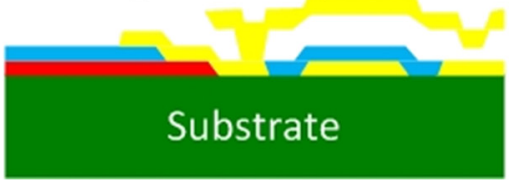

Fig. 2 Microfabrication process 

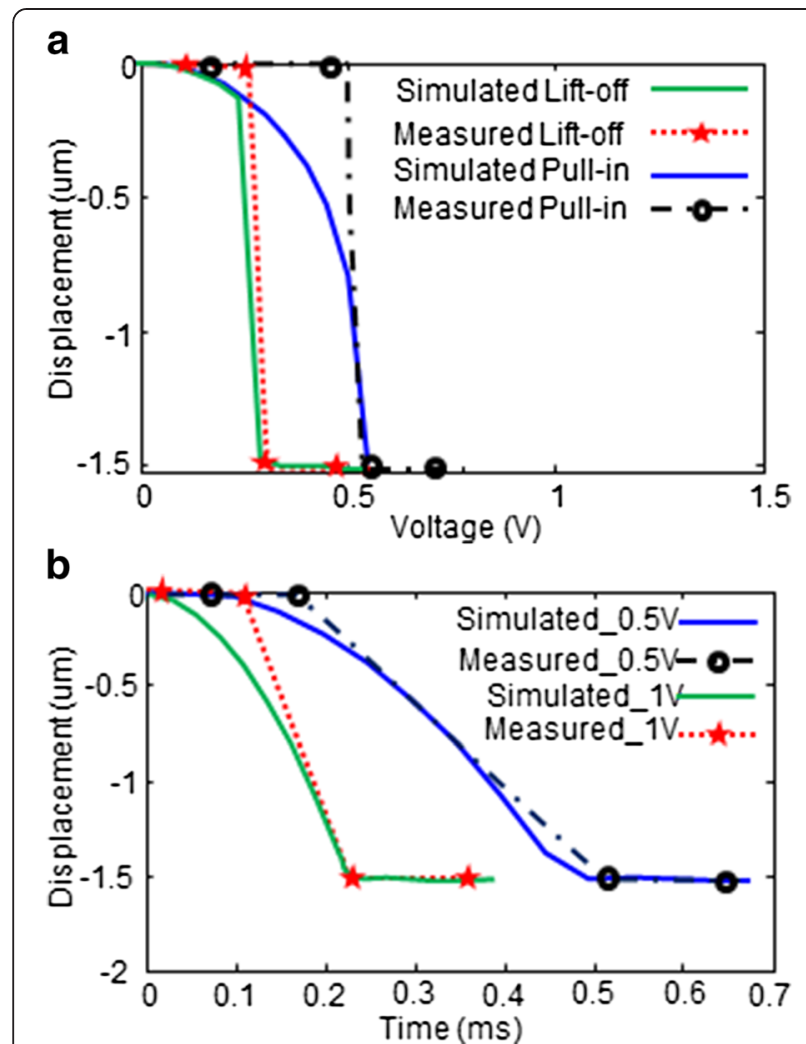

Fig. 3 a Pull-in and lift-off voltages of the proposed MEMS switch, (b) Displacement vs time in Z direction with different applied voltages

and $L_{s}$, respectively. $W$ and $t$ are width and thickness of the cantilever.

Current distribution is high on the edges of the cantilever beam. In the proposed design, some small holes are made in the beam to increase the effective area of the edges to increase the speed of the switching. Helix restoring spring with length and width of $700 \mu \mathrm{m}$ and $30 \mu \mathrm{m}$ are chosen to support the low actuation voltage with a small area. Actuation pad length and width are designed to be $190 \mu \mathrm{m}$ and $220 \mu \mathrm{m}$. The Helix high resistivity traces (HRT) provides enough resistivity to isolate the input signal from the DC supply. The gap between dimple and transmission line is optimized to provide enough isolation in the OFF state mode. A $12.5 \mu \mathrm{m} \times 25 \mu \mathrm{m}$ dimple is chosen to meet enough isolation in the OFF state and low contact resistance in the ON state for RF applications. The fabrication process allows the reduction of the gap between metal plates, $g_{0}$, down to $1.5 \mu \mathrm{m}$. A thinner cantilever beam reduces the spring constant and consequently lowers the actuation voltage but it also reduces the weight which is inversely proportional to the actuation voltage. It will also increase the pull-in switching speed since the pull-in acceleration of the movable actuator is inversely proportional to its mass. The transient time for lift-off increases with the

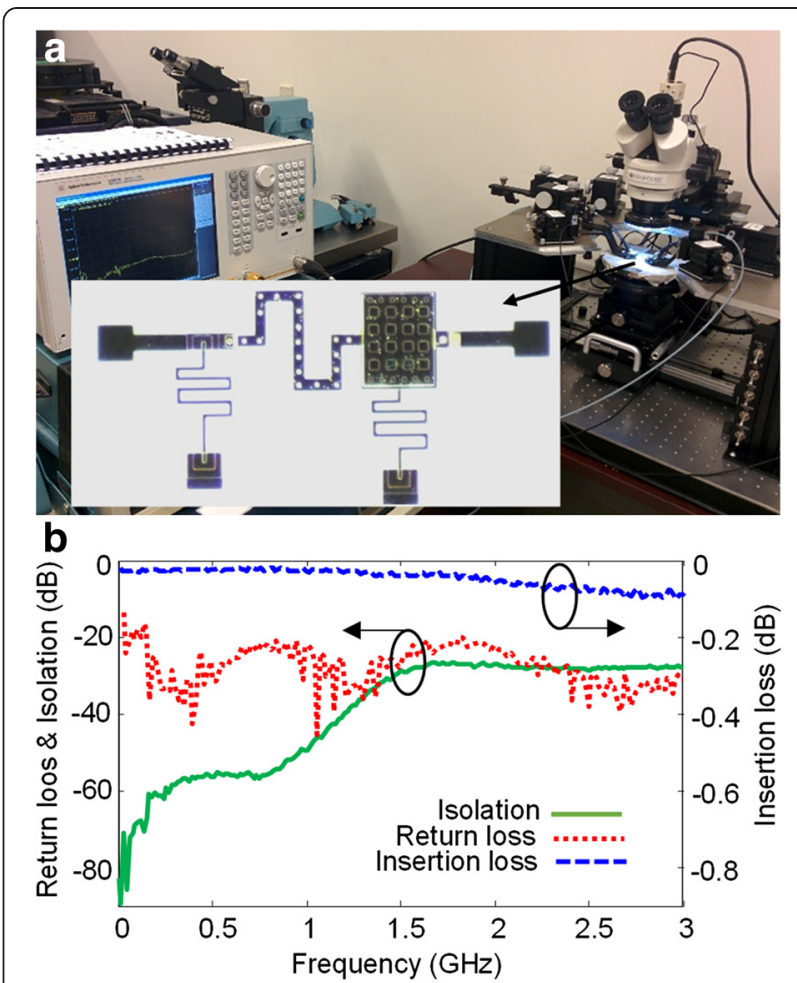

Fig. 4 a Prototype under test using Agilent E5061B network analyzer and MA-8100 RF probes from SEMIPROBE ${ }^{\mathrm{TM}}$, (b) Return loss, insertion loss and isolation versus frequency

reduction of the movable actuator thickness due to the electrostatic capacitor hysteresis. An optimization for pull-in and lift-off voltages, resulted in a $2 \mu \mathrm{m}$ thick movable actuator. Figure 1, presents 3D view of the implemented low actuation voltage using Coventorware ${ }^{\mathrm{Tx}}$. The total fabrication area of the switch is $1264 \mu \mathrm{m} \times 635 \mu \mathrm{m}$, including input and output transmission lines, DC bias lines and pads.

Table 1 Comparison of key features

\begin{tabular}{|c|c|c|c|}
\hline Parameter & Ref. [5] & Ref. [6] & This paper \\
\hline Frequency (GHz) & 50 & 2 & $0-3$ \\
\hline Pull-in Voltage & 4.5 & 15 & 0.5 \\
\hline Displacement $(\mu \mathrm{m})$ & 1.5 & 1.3 & 1.5 \\
\hline Transition Time & $120 \mathrm{~ns}$ & $7.4 \mu \mathrm{s}$ & $0.5 \mathrm{~ms}$ \\
\hline Isolation (dB) & 55 & 45.7 & 27 \\
\hline Insertion loss (dB) & 0.5 & 0.25 & 0.1 \\
\hline Return loss (dB) & 12.4 & NA & 20 \\
\hline $\begin{array}{l}1 \mathrm{~V} \text { CMOS } \\
\text { technology }\end{array}$ & No & No & Yes \\
\hline Life Cycle & $8 \times 10^{8}$ & $1 \times 10^{7}$ & $1 \times 10^{4}$ \\
\hline \multirow[t]{2}{*}{ Size } & $400 \times 100 \mu \mathrm{m}^{2}$ & $2000 \times 1000 \mu \mathrm{m}^{2}$ & $1264 \times 635 \mu \mathrm{m}^{2}$ \\
\hline & $\begin{array}{l}\text { (Lower contact } \\
\text { pad) }\end{array}$ & (SPDT) & $\begin{array}{l}\text { (Including } \\
\text { biasing pads) }\end{array}$ \\
\hline
\end{tabular}




\section{Fabrication process}

A prototypes is fabricated using a cost effective microfabrication process as a proof of concept. The fabrication process employs eight steps, as shown in Fig. 2. The substrate is a $635 \mu \mathrm{m}$ thick Alumina with loss tangent of 0.0001 at $1 \mathrm{MHz}$ and relative permittivity of 9.9. Electron beam-write chromium masks are used during the fabrication process. At the first step, a $50 \mathrm{~nm}$ Titanium tungsten (TiW) is sputtered and patterned. This creates HRT to connect the DC bias pads to the actuation pad and input signal line. The first step is followed by deposition of $0.7 \mu \mathrm{m} \mathrm{SiO}_{2}$ at $250^{\circ} \mathrm{C}$ by a plasma enhanced chemical vapor deposition (PECVD) and patterned using reactive ion etching (RIE). Then a seed layer is deposited by evaporation of $40 \mathrm{~nm} \mathrm{Cr} / 70 \mathrm{~nm}$ Au bilayer. G1 is patterned using a negative photoresist mold by photolithography process. This is followed by electroplating $1 \mu \mathrm{m}$ $\mathrm{Au}$ within the mold.

The $\mathrm{Cr}$ layer is used as an adhesion layer for Au which is washed out later. The fabrication process is followed by $0.7 \mu \mathrm{m} \mathrm{SiO}_{2}$ PECVD deposition at $250{ }^{\circ} \mathrm{C}$ as dielectric. A $30 \mathrm{~nm}$ TiW is used at this step as an adhesion layer between D2 and G1. In step five a $2.5 \mu \mathrm{m}$ thick spin coated polyimide layer is used as a sacrificial layer. To create the anchor and the dimple holes, polyimide is patterned using RIE. Then the fabrication process is completed by sputtering the $70 \mathrm{~nm} \mathrm{Au}$ as a seed layer and an electroplated $\mathrm{Au}$ layer with total thickness of $2 \mu \mathrm{m}$ and removing the sacrificial layer using $\mathrm{O}_{2}$ plasma dry etching in RIE.

\section{Simulation and measurement results}

Figure 3a shows the pull-in and lift-off voltages of $0.5 \mathrm{~V}$ and $0.3 \mathrm{~V}$ with $1.5 \mu \mathrm{m}$ displacement using the finite element analysis (FEA) tools in Coventorware ${ }^{\mathrm{Tt}}$ and they are in agreement with measurement results. The transient response in Fig. 3b, indicates that $0.5 \mathrm{~ms}$ is required for switching at the pull-in voltage of $0.5 \mathrm{~V}$. It can be seen that by increasing the applied voltage, the switching time reduces. The number of switching cycles of this switch has reached $1 \times 10^{4}$ cycles at $0.5 \mathrm{v}$. Figure 4 a shows the fabricated MEMS switch under test using MA-8100 RF probes from Semiprobe ${ }^{\mathrm{Th}}$ and an Agilent E5061B $3 \mathrm{kHz}$ to $3 \mathrm{GHz}$ ENA network analyzer. Figure $4 \mathrm{~b}$ shows $\mathrm{S}$ parameter measurement results for the MEMS switch at the ON and OFF states. An insertion loss of less than $-0.1 \mathrm{~dB}$ and return loss of better than $-20 \mathrm{~dB}$ were measured over the entire frequency range of $100 \mathrm{kHz}$ to $3 \mathrm{GHz}$. The switch also shows an isolation of higher than $-27 \mathrm{~dB}$ over the measured frequency range as shown in Fig. 3b. Table 1 presents a comparison between the key features of the designed MEMS switch with reported works in the literature $[5,6]$.

\section{Conclusion}

This paper presents a new low actuation voltage RF MEMS switch. A helix restoring spring together with a moving plate containing square holes are used to lower the pull-in voltage. A prototype is fabricated as a proof of concept using a fabrication process with seven electronbeam-write chromium masks. The switch is designed and optimized using Coventorware ${ }^{\mathrm{Tw}}$. Measurement results indicate a pull-in voltage of $0.5 \mathrm{~V}$ which makes the switch ideal for integration with available low voltage CMOS technologies. It also presents $-0.1 \mathrm{~dB}$ insertion loss and less than $-20 \mathrm{~dB}$ return loss over a frequency range extending from $3 \mathrm{kHz}$ to $3 \mathrm{GHz}$.

\section{Abbreviations}

RF: Radio frequency; MEMS: Microelectromechanical systems; CMOS: Complementary metal-oxide semiconductor; HRT: High resistivity traces; TiW: Titanium tungsten; PECVD: Plasma enhanced chemical vapor deposition; RIE: Reactive ion etching; FEA: Finite element analysis.

\section{Competing interests}

The authors declare that they have no competing interests.

\section{Authors' contributions}

AA participated in design, simulation, fabrication and test the device and helped to draft the manuscript and analyzed the data. RM and RR conceived and supervised this study. All authors read and approved the final manuscript.

\section{Acknowledgment}

The authors would like to thank the research and financial supports received from Natural Sciences and Engineering Research Council (NSERC) of Canada and CMC Microsystems.

Received: 6 May 2015 Accepted: 4 August 2015

Published online: 11 August 2015

\section{References}

1. Attar SS, Setoodeh S, Mansour RR, Gupta D (2014) Low-Temperature Superconducting DC-Contact RF MEMS Switch for Cryogenic Reconfigurable RF Front-Ends. IEEE Trans Microw Theory Tech 62:1437-1447. doi:10.1109/TMTT.2014.2327205

2. Rajagopalan H, Kovitz JM, Rahmat-Samii Y (2014) MEMS Reconfigurable Optimized E-Shaped Patch Antenna Design for Cognitive Radio. IEEE Trans Antennas Propag 62:1056-1064. doi:10.1109/TAP.2013.2292531

3. Daneshmand M (2006) Multi-Port RF MEMS Switches and Switch Matrices. Dissertation, University of Waterloo. http://hdl.handle.net/10012/878

4. Rebeiz GM (2003) RF MEMS: Theory, Design, and Technology. Wiley, Hoboken, NJ

5. Lee SD, Jun BC, Kim SD, Rhee JK (2005) A novel pull-up type RF MEMS switch with low actuation voltage. IEEE Microw Compon Lett 15:856-858. doi:10.1109/LMWC.2005.860006

6. Kim CH (2012) Mechanically Coupled Low-Voltage Electrostatic Resistive RF Multithrow Switch. IEEE Trans Ind Electron 59:1114-1122. doi:10.1109/ TIE.2011.2159694 